\title{
Dual variational methods for a nonlinear Helmholtz equation with sign-changing nonlinearity
}

\author{
Rainer Mandel ${ }^{1}$ - Dominic Scheider ${ }^{1} \cdot$ Tolga Yeşil $^{2}$
}

Received: 14 January 2021 / Accepted: 26 March 2021 / Published online: 29 June 2021

(c) The Author(s) 2021

\section{Abstract}

We prove new existence results for a nonlinear Helmholtz equation with sign-changing nonlinearity of the form

$$
-\Delta u-k^{2} u=Q(x)|u|^{p-2} u, \quad u \in W^{2, p}\left(\mathbb{R}^{N}\right)
$$

with $k>0, N \geq 3, p \in\left[\frac{2(N+1)}{N-1}, \frac{2 N}{N-2}\right)$ and $Q \in L^{\infty}\left(\mathbb{R}^{N}\right)$. Due to the sign-changes of $Q$, our solutions have infinite Morse-Index in the corresponding dual variational formulation.

Mathematics Subject Classification 35J20 $\cdot 35 \mathrm{Q} 60$

\section{Introduction}

Real-valued solutions of nonlinear Helmholtz equations of the form

$$
-\Delta u-k^{2} u=g\left(x,|u|^{2}\right) u, \quad x \in \mathbb{R}^{N}
$$

arise in the study of high-frequency time-harmonic solutions of the Klein-Gordon equation

$$
\frac{\partial^{2} U}{\partial t^{2}}-\Delta U+m U=g\left(x,|U|^{2}\right) U, \quad m>0, \quad(t, x) \in \mathbb{R} \times \mathbb{R}^{N}
$$

which is an important model in the mathematical description of nonlinear wave propagation. Indeed, plugging in the time-harmonic ansatz $U(t, x)=e^{\mathrm{i} \omega t} u(x)$ for $\omega^{2}>m$ we find that the real-valued profile function $u: \mathbb{R}^{N} \rightarrow \mathbb{R}$ satisfies (1.1) with $k^{2}=\omega^{2}-m>0$. In other words, nonlinear Helmholtz equations can be seen as the high-frequency counterparts of the well-studied class of stationary nonlinear Schrödinger equations where $\omega^{2}<m$ is assumed.

Communicated by E. Lenzmann.

Rainer Mandel

Rainer.Mandel@kit.edu

1 Karlsruhe Institute of Technology, Karlsruhe, Germany

2 Goethe University Frankfurt, Karlsruhe, Germany 
In the present article, we consider nonlinear Helmholtz equations of the form

$$
-\Delta u-k^{2} u=Q(x)|u|^{p-2} u \quad \text { on } \mathbb{R}^{N}
$$

for $p \in\left[\frac{2(N+1)}{N-1}, \frac{2 N}{N-2}\right)$ and $k>0$ with a weight function $Q \in L^{\infty}\left(\mathbb{R}^{N}\right)$ that may change sign. One of the main tools for proving the existence of solutions is the dual variational method that, in its classical form, relies on the nonnegativity of the potential $Q$. In the context of Nonlinear Helmholtz equations this method was first implemented in a paper by Evéquoz and Weth [4]. To highlight the role of the nonnegativity of $Q$ we briefly recapitulate the approach.

Instead of (1.2) one considers a reformulation as the integral equation

$$
u=\mathbf{R}\left(Q|u|^{p-2} u\right) \quad u \in L^{p}\left(\mathbb{R}^{N}\right),
$$

where $\mathbf{R}$ is the real part of a resolvent type operator $\mathcal{R}$, i.e., a right inverse of the Helmholtz operator $-\Delta-k^{2}$ on $\mathbb{R}^{N}$. For $f \in \mathcal{S}\left(\mathbb{R}^{N}\right)$ the operator $\mathcal{R}$ is given by $\mathcal{R}(f)=\Phi * f$ where

$$
\Phi(x):=\frac{\mathrm{i}}{4}\left(\frac{k}{2 \pi|x|}\right)^{\frac{N-2}{2}} H_{\frac{N-2}{2}}^{(1)}(k|x|), \quad x \in \mathbb{R}^{N} \backslash\{0\}
$$

is the fundamental solution of the Helmholtz equation associated with Sommerfeld's outgoing radiation condition

$$
\left|\nabla \Phi(x)-k \mathrm{i} \Phi(x) \frac{x}{|x|}\right|=o\left(|x|^{\frac{1-N}{2}}\right), \quad \text { as }|x| \rightarrow \infty .
$$

Here, $H_{\frac{N-2}{2}}^{(1)}$ denotes the Hankel function of the first kind and order $\frac{N-2}{2}$. So the operator $\mathbf{R}$ from (1.3) is given by $R(f)=\Psi * f$ where $\Psi:=\operatorname{Re}(\Phi)$ satisfies

$$
\Psi(x)=-\frac{1}{4}\left(\frac{k}{2 \pi|x|}\right)^{\frac{N-2}{2}} Y_{\frac{N-2}{2}}(k|x|), \quad x \in \mathbb{R}^{N} \backslash\{0\} .
$$

The symbol $Y_{\frac{N-2}{2}}=\operatorname{Im}\left(H_{\frac{N-2}{2}}^{(1)}\right)$ stands for the Bessel function of the second kind of order $\frac{N-2}{2}$. It is known [7, Theorem 2.3] that $\mathcal{R}$ extends as a continuous linear map from $L^{p^{\prime}}\left(\mathbb{R}^{N}\right) \rightarrow L^{p}\left(\mathbb{R}^{N}\right)$ precisely for $p \in\left[\frac{2(N+1)}{N-1}, \frac{2 N}{N-2}\right]$. To explain this briefly notice that the Fourier symbol of $\mathcal{R}$ is the tempered distribution $\left(|\xi|^{2}-k^{2}-i 0\right)^{-1}$. Its behaviour at infinity $\sim|\xi|^{-2}$ is responsible for the upper bound $\frac{2 N}{N-2}$ whereas frequencies $|\xi| \approx k$ give rise to the lower bounds given by the Stein-Tomas exponent $p_{S T}:=\frac{2(N+1)}{N-1}$. Recall that the Stein-Tomas inequality reads

$$
\|\hat{f}\|_{L^{2}\left(S^{N-1}\right)} \leq\|f\|_{L^{\frac{2(N+1)}{N+3}}\left(\mathbb{R}^{N}\right)}, \quad N \geq 2 .
$$

Here, $S^{N-1}$ denotes the unit sphere in $\mathbb{R}^{N}$ and $\frac{2(N+1)}{N+3}=p_{S T}^{\prime}$ is the dual Stein-Tomas exponent.

Being given these mapping properties of $\mathcal{R}$ and hence of $\mathbf{R}=\operatorname{Re}(\mathcal{R})$ we introduce the dual variable $\tilde{u}:=Q^{1 / p^{\prime}}|u|^{p-2} u$ and observe that solutions of (1.3) are precisely the critical points of the (dual) energy functional $I: L^{p^{\prime}}\left(\mathbb{R}^{N}\right) \rightarrow L^{p}\left(\mathbb{R}^{N}\right)$ given by

$$
I(\tilde{u}):=\frac{1}{p^{\prime}}\|\tilde{u}\|_{p^{\prime}}^{p^{\prime}}-\frac{1}{2} \int_{\mathbb{R}^{N}} \tilde{u} \mathcal{K} \tilde{u} d x .
$$


Here, $\mathcal{K}: L^{p^{\prime}}\left(\mathbb{R}^{N}\right) \rightarrow L^{p}\left(\mathbb{R}^{N}\right), \tilde{u} \mapsto Q^{\frac{1}{p}} \mathbf{R}\left(Q^{\frac{1}{p}} \tilde{u}\right)$ is a symmetric operator in the sense of

$$
\int_{\mathbb{R}^{N}} f \mathcal{K} g d x=\int_{\mathbb{R}^{N}} g \mathcal{K} f d x \quad \text { for all } f, g \in L^{p^{\prime}}\left(\mathbb{R}^{N}\right),
$$

see [4, Lemma 4.1]. We point out that the proof of this fact uses that $Q$ is nonnegative. Under the additional assumption that $Q$ vanishes at infinity, one obtains that $I$ is an odd functional of class $\mathcal{C}^{1}$ that has the Mountain Pass Geometry and satisfies the Palais-Smale Condition. So the existence of an unbounded sequence of solutions to (1.3) follows from the Symmetric Mountain Pass Theorem. Inverting the transformation $u \mapsto \tilde{u}$ one thus obtains an unbounded sequence of solutions to the nonlinear Helmholtz equation (1.2). This is the strategy proposed by Evéquoz and Weth [4] for the focusing nonlinear Helmholtz equation where $Q \geq 0$. We refer to [9] for analogous results in the defocusing case $Q \leq 0$, where the dual variational approach was implemented for the dual variable $\tilde{u}:=|Q|^{1 / p^{\prime}}|u|^{p-2} u$. In view of these two results it is natural to ask for a dual variational approach work in the intermediate case of sign-changing $Q$. In this paper, we provide a solution for this problem.

To treat sign-changing coefficients $Q \in L^{\infty}\left(\mathbb{R}^{N}\right)$ we have to come up with a new idea to make the dual variational approach work. We write $Q=Q_{+}-Q_{-}$where $Q_{ \pm}:=|Q| \mathbb{1}_{A_{ \pm}}$ and

$$
A_{+}:=\{Q>0\}, \quad A_{-}:=\{Q \leq 0\} .
$$

In fact we will consider $Q_{\lambda}:=\lambda Q_{+}-Q_{-}$for $\lambda>0$ in the following. Our main idea is to introduce the dual variables $(\phi, \psi) \in L^{p^{\prime}}\left(A_{+}\right) \times L^{p^{\prime}}\left(A_{-}\right)$associated with $\left(\left.u\right|_{A_{+}},\left.u\right|_{A_{-}}\right)$ and to derive a coupled system of nonlinear integral equations the solutions of which are precisely the critical points of an associated strongly indefinite dual energy functional. We will see that the indefiniteness comes from the presence of $Q_{-}$and thus vanishes in the case of a nonnegative function $Q \geq 0$. In particular, the critical points of this dual energy functional will have infinite Morse index, which clearly distinguishes these solutions from the dual bound and ground states obtained in [4] in the case $Q \geq 0$. We will explain the dual variational framework in detail in Sect. 2. Our conditions for the existence of critical points involve the linear operator $\mathcal{K}: L^{p^{\prime}}\left(\mathbb{R}^{N}\right) \rightarrow L^{p}\left(\mathbb{R}^{N}\right), f \mapsto|Q|^{\frac{1}{p}} \mathbf{R}\left(|Q|^{\frac{1}{p}} f\right)$ as well as the numbers

$$
\alpha:=\max _{\substack{\|\phi\|_{p^{\prime}}=1, \operatorname{supp}(\phi) \subset A_{+}}} \int_{\mathbb{R}^{N}} \phi \mathcal{K} \phi d x, \quad \beta:=\max _{\begin{array}{c}
\|\phi\|_{p^{\prime}}=\|\psi\|_{p^{\prime}}=1, \\
\operatorname{supp}(\phi) \subset A_{+}, \operatorname{supp}(\psi) \subset A_{-}
\end{array}} \int_{\mathbb{R}^{N}} \phi \mathcal{K} \psi d x .
$$

Since we will assume $\mathcal{K}$ to be compact, both values are indeed attained. Moreover we have $\beta \geq 0$ and [4, Lemma 4.2(ii)] gives $\alpha>0$ once we assume that $A^{+}$has positive measure, i.e., $Q^{+} \not \equiv 0$. Our main result reads as follows.

Theorem 1.1 Let $p \in\left[\frac{2(N+1)}{N-1}, \frac{2 N}{N-2}\right)$ and $Q \in L^{\infty}\left(\mathbb{R}^{N}\right), Q^{+} \not \equiv 0$. Moreover assume that

$$
\mathcal{K}: L^{p^{\prime}}\left(\mathbb{R}^{N}\right) \rightarrow L^{p}\left(\mathbb{R}^{N}\right) \text { is compact and } \int_{\mathbb{R}^{N}} \psi \mathcal{K} \psi d x \geq 0 \text { for all } \psi \in L^{p^{\prime}}\left(A_{-}\right)(1.9)
$$

Then for almost all $\lambda>\lambda_{0}:=\left(2 \beta \alpha^{-1}\right)^{p}$ there is a nontrivial strong solution $u \in W^{2, q}\left(\mathbb{R}^{N}\right) \cap$ $\mathcal{C}^{1, \gamma}\left(\mathbb{R}^{N}\right)$ for all $q \in\left(\frac{2 N}{N-1}, \infty\right), \gamma \in(0,1)$ of

$$
-\Delta u-k^{2} u=Q_{\lambda}(x)|u|^{p-2} u \quad \text { on } \mathbb{R}^{N} \text {. }
$$


The proof relies on a combination of a saddle-point reduction and the abstract monotonicity trick by Jeanjean-Toland [6], which provides bounded Palais-Smale sequences (only) for almost all $\lambda>\lambda_{0}$. It would clearly be desirable to extend our result to all $\lambda>\lambda_{0}$, but related a priori bounds seem to be out of reach. Notice also that [9, Theorem 1.4] suggests the existence of nontrivial solutions also for small $\lambda>0$, possibly assuming the set $\{Q>0\}$ to be small enough and following a different variational approach. Let us point out that $\lambda_{0}$ is small provided that the number $\beta$, which is the same as the operator norm $\left\|\mathbb{1}_{A_{+}} \mathcal{K}\left(\mathbb{1}_{A_{-}}\right)\right\|_{p^{\prime} \rightarrow p}$, is small compared to $\alpha>0$. In the case $p>\frac{2(N+1)}{N-1}$ this can be achieved by considering coefficient functions $Q$ such that $\operatorname{dist}\left(A_{-}, A_{+}\right)$is large enough, see [5, Lemma 2.6].

In the following Corollary, we show that the abstract conditions (1.9) hold for a reasonable class of sign-changing functions $Q \in L^{\infty}\left(\mathbb{R}^{N}\right)$. If for instance $Q$ vanishes at infinity, then [4, Lemma 4.1(ii)] applied to $|Q|$ implies that $\mathcal{K}: L^{p^{\prime}}\left(\mathbb{R}^{N}\right) \rightarrow L^{p}\left(\mathbb{R}^{N}\right)$ is compact. It is less immediate to verify the non-negativity assumption on the bilinear form (1.9). From [2, Corollary 5.4] we infer that this condition holds for measurable sets $A_{-}$with small enough diameter. To be more precise, if $y_{\frac{N-2}{2}}$ denotes the first (positive) zero of the Bessel function $Y_{\frac{N-2}{2}}$, then the condition $\operatorname{diam}\left(A_{-}\right) \leq k^{-1} y_{\frac{N-2}{2}}$ is sufficient. To put this condition into perspective, note that for $N=3$ we have $Y_{\frac{1}{2}}(t)=-\sqrt{\frac{2}{\pi t}} \cos t$, thus $y_{1 / 2}=\pi / 2$ and $y_{\frac{N-2}{2}}>y_{1 / 2}$ for $N>3$ (see [1], [Section 9.5]). We thus conclude as follows.

Corollary 1.2 Assume $p \in\left[\frac{2(N+1)}{N-1}, \frac{2 N}{N-2}\right)$ and $Q \in L^{\infty}\left(\mathbb{R}^{N}\right), Q^{+} \not \equiv 0$. Moreover assume

$$
\lim _{R \rightarrow \infty} \operatorname{esssup}_{|x| \geq R}|Q(x)|=0 \quad \text { and } \quad \operatorname{diam}\left(A_{-}\right) \leq k^{-1} y_{\frac{N-2}{2}} .
$$

Then for almost all $\lambda>\lambda_{0}:=\left(2 \beta \alpha^{-1}\right)^{p}$ there is a nontrivial strong solution $u \in$ $W^{2, q}\left(\mathbb{R}^{N}\right) \cap \mathcal{C}^{1, \gamma}\left(\mathbb{R}^{N}\right)$ for all $q \in\left(\frac{2 N}{N-1}, \infty\right)$ and $\gamma \in(0,1)$ of $(1.10)$.

The regularity results in Theorem 1.1 and Corollary 1.2 are direct consequences of [4, Lemma 4.3] and of the iteration procedure from Step 3 in the proof of [8, Theorem 1]. Notice that [8, Theorem 1] provides solutions to far more general Nonlinear Helmholtz equations than (1.2) regardless of whether sign-changes occur or not, but the constructed solutions are small. This result relies on a fixed point approach. Let us also mention [3] where nontrivial solutions of Nonlinear Helmholtz equations are constructed for rather general and possibly sign-changing nonlinearities vanishing identically outside some compact subset of $\mathbb{R}^{N}$. Our method is entirely different from any of these approaches.

This paper is organzied as follows: In Sect. 2 we introduce our basic tools and develop the dual variational framework by reformulating the indefinite Nonlinear Helmholtz equation as a coupled system of integral equations. Then we prove that nontrivial critical points of the associated energy functional $J_{\lambda}$ are indeed nontrivial solutions $u \in L^{p}\left(\mathbb{R}^{N}\right)$ of the integral equation $u=\mathbf{R}\left(Q_{\lambda}|u|^{p-2} u\right)$. This motivates the search for critical points of $J_{\lambda}$. In Sect. 3 we perform the saddle-point reduction of $(\phi, \psi) \mapsto J_{\lambda}(\phi, \psi)$ leading to a reduced function $\tilde{J}_{\lambda}$ that depends on $\phi$ only. In Sect. 4 we establish the existence of bounded Palais-Smale sequences for these reduced functionals for almost all $\lambda>\lambda_{0}$. As mentioned above, this step entirely relies on the monotonicity trick by Jeanjean and Toland [6]. Finally, we combine all the auxiliary results to prove Theorem 1.1 and Corollary 1.2 in Sect. 5.

Let us close this introduction by fixing some notation: Throughout the paper we denote by $B_{r}(x)$ the open ball in $\mathbb{R}^{N}$ with radius $r>0$ and center at $x \in \mathbb{R}^{N}$. Moreover, we set $B_{r}=B_{r}(0)$ for any $r>0$. For $1 \leq s \leq \infty$, we abbreviate the standard norm on $L^{s}\left(\mathbb{R}^{N}\right)$ by $\|\cdot\|_{s}$. The Schwartz-class of rapidly decreasing functions on $\mathbb{R}^{N}$ is denoted by $\mathcal{S}\left(\mathbb{R}^{N}\right)$. For 
any $p \in(1, \infty)$ we always denote by $p^{\prime}:=\frac{p}{p-1}$ the Hölder conjugate of $p$. The indicator function of a measurable set $B \subset \mathbb{R}^{N}$ is $\mathbb{1}_{B}$. By diam $(B)$ we always denote the diameter of a set. We will always use the symbols $\phi, \psi$ to denote $L^{p^{\prime}}\left(A_{+}\right)-$and $L^{p^{\prime}}\left(A_{-}\right)$-functions that are continued trivially to the whole of $\mathbb{R}^{N}$.

\section{Dual variational formulation}

In this section we will formulate a variational framework to the Eq. (1.10). We recall from the introduction that solutions of our problem are obtained as solutions of the integral equation

$$
u=\mathbf{R}\left(Q_{\lambda}|u|^{p-2} u\right), \quad u \in L^{p}\left(\mathbb{R}^{N}\right) .
$$

where $\mathbf{R}(f)=\Psi * f$ for the function $\Psi$ introduced in (1.5) and

$$
\|\mathbf{R}(f)\|_{L^{p}\left(\mathbb{R}^{N}\right)} \leq C\|f\|_{L^{p^{\prime}}\left(\mathbb{R}^{N}\right)}
$$

for all $p \in\left[\frac{2(N+1)}{N-1}, \frac{2 N}{N-2}\right]$ and some constant $C>0$.

To obtain the dual variational formulation of (2.1) we introduce $v:=\mathbb{1}_{A_{+}} u$ and $w:=\mathbb{1}_{A_{-}} u$. Then (2.1) is equivalent to the system

$$
\begin{aligned}
v & =\lambda \mathbb{1}_{A_{+}} \mathbf{R}\left[Q_{+}|v|^{p-2} v\right]-\mathbb{1}_{A_{+}} \mathbf{R}\left[Q_{-}|w|^{p-2} w\right], \\
w & =\lambda \mathbb{1}_{A_{-}} \mathbf{R}\left[Q_{+}|v|^{p-2} v\right]-\mathbb{1}_{A_{-}} \mathbf{R}\left[Q_{-}|w|^{p-2} w\right] .
\end{aligned}
$$

Setting

$$
\phi:=\lambda Q_{+}^{1 / p^{\prime}}|v|^{p-2} v \in L^{p^{\prime}}\left(A_{+}\right), \quad \psi:=Q_{-}^{1 / p^{\prime}}|w|^{p-2} w \in L^{p^{\prime}}\left(A_{-}\right)
$$

we deduce

$$
\begin{aligned}
\lambda^{1-p^{\prime}}|\phi|^{p^{\prime}-2} \phi & =Q_{+}^{1 / p} v \\
& =\lambda Q_{+}^{1 / p} \mathbf{R}\left[Q_{+}|v|^{p-2} v\right]-Q_{+}^{1 / p} \mathbf{R}\left[Q_{-}|w|^{p-2} w\right] \\
& =Q_{+}^{1 / p} \mathbf{R}\left[Q_{+}^{1 / p} \phi\right]-Q_{+}^{1 / p} \mathbf{R}\left[Q_{-}^{1 / p} \psi\right] \\
& =Q_{+}^{1 / p} \mathbf{R}\left[|Q|^{1 / p}(\phi-\psi)\right] .
\end{aligned}
$$

Similarly

$$
|\psi|^{p^{\prime}-2} \psi=Q_{-}^{1 / p} \mathbf{R}\left[|Q|^{1 / p}(\phi-\psi)\right]
$$

In terms of the Birman-Schwinger operator $\mathcal{K}: f \mapsto|Q|^{\frac{1}{p}} \mathbf{R}\left(|Q|^{\frac{1}{p}} f\right)$ introduced above this can be reformulated as

$$
\begin{aligned}
\lambda^{1-p^{\prime}}|\phi|^{p^{\prime}-2} \phi & =\mathbb{1}_{A_{+}} \mathcal{K}(\phi-\psi), \\
|\psi|^{p^{\prime}-2} \psi & =\mathbb{1}_{A_{-}} \mathcal{K}(\phi-\psi)
\end{aligned}
$$

and therefore carries a variational structure through the (dual) energy functional $J_{\lambda}$ on $L^{p^{\prime}}\left(A_{+}\right) \times L^{p^{\prime}}\left(A_{-}\right)$given by

$$
J_{\lambda}(\phi, \psi):=\frac{\lambda^{1-p^{\prime}}}{p^{\prime}}\|\phi\|_{p^{\prime}}^{p^{\prime}}-\frac{1}{p^{\prime}}\|\psi\|_{p^{\prime}}^{p^{\prime}}-\frac{1}{2} \int_{\mathbb{R}^{N}}(\phi-\psi) \mathcal{K}(\phi-\psi) d x .
$$


This functional is of class $\mathcal{C}^{1}$ with

$$
\begin{aligned}
& \partial_{1} J_{\lambda}(\phi, \psi)\left[h_{1}\right]=\int_{\mathbb{R}^{N}}\left(\lambda^{1-p^{\prime}}|\phi|^{p^{\prime}-2} \phi-\mathcal{K}(\phi-\psi)\right) h_{1} d x, h_{1} \in L^{p^{\prime}}\left(A_{+}\right) \\
& \partial_{2} J_{\lambda}(\phi, \psi)\left[h_{2}\right]=\int_{\mathbb{R}^{N}}\left(-|\psi|^{p^{\prime}-2} \psi-\mathcal{K}(\phi-\psi)\right) h_{2} d x, h_{2} \in L^{p^{\prime}}\left(A_{-}\right) .
\end{aligned}
$$

Here $\partial_{1}, \partial_{2}$ stand for partial derivatives with respect to $\phi$ and $\psi$. For this reason we will look for critical points of $J_{\lambda}$. These solve the integral equation (2.1). Thus by the regularity results [4, Lemma 4.3] and [8, p.13] these are indeed strong solutions to our original problem (1.10).

Proposition 2.1 Let $(\phi, \psi) \in L^{p^{\prime}}\left(A_{+}\right) \times L^{p^{\prime}}\left(A_{-}\right) \backslash\{(0,0)\}$ be a critical point of $J_{\lambda}$ where $\lambda>0$. Then

$$
u:=\mathbf{R}\left(|Q|^{\frac{1}{p}}(\phi-\psi)\right) \in L^{p}\left(\mathbb{R}^{N}\right)
$$

is a nontrivial solution of (2.1).

Proof Let $(\phi, \psi) \in L^{p^{\prime}}\left(A_{+}\right) \times L^{p^{\prime}}\left(A_{-}\right) \backslash\{(0,0)\}$ be a critical point of $J_{\lambda}$. Thus we have

$$
\lambda^{1-p^{\prime}}|\phi|^{p^{\prime}-2} \phi=\mathbb{1}_{A_{+}} \mathcal{K}(\phi-\psi), \quad|\psi|^{p^{\prime}-2} \psi=\mathbb{1}_{A_{-}} \mathcal{K}(\phi-\psi)
$$

as well as

$$
\begin{aligned}
Q_{\lambda}|u|^{p-2} u & =\left(\lambda \mathbb{1}_{A_{+}}-\mathbb{1}_{A_{-}}\right)|Q||u|^{p-2} u \\
& =\left.\left.\left(\lambda \mathbb{1}_{A_{+}}-\mathbb{1}_{A_{-}}\right)|Q|^{\frac{1}{p}} \cdot|| Q\right|^{\frac{1}{p}} u\right|^{p-2} \cdot|Q|^{\frac{1}{p}} u \\
& =\left.\left.\left(\lambda \mathbb{1}_{A_{+}}-\mathbb{1}_{A_{-}}\right)|Q|^{\frac{1}{p}} \cdot|| Q\right|^{\frac{1}{p}} \mathbf{R}\left[|Q|^{1 / p}(\phi-\psi)\right]\right|^{p-2} \cdot|Q|^{\frac{1}{p}} \mathbf{R}\left[|Q|^{1 / p}(\phi-\psi)\right] \\
& =\left(\lambda \mathbb{1}_{A_{+}}-\mathbb{1}_{A_{-}}\right)|Q|^{\frac{1}{p}} \cdot|\mathcal{K}[\phi-\psi]|^{p-2} \cdot \mathcal{K}[\phi-\psi] \\
& =\left.\left(\lambda \mathbb{1}_{A_{+}}-\mathbb{1}_{A_{-}}\right)|Q|^{\frac{1}{p}} \cdot\left|\lambda^{1-p^{\prime}}\right| \phi\right|^{p^{\prime}-2} \phi+\left.|\psi|^{p^{\prime}-2} \psi\right|^{p-2} \cdot\left(\lambda^{1-p^{\prime}}|\phi|^{p^{\prime}-2} \phi+|\psi|^{p^{\prime}-2} \psi\right) \\
& =\left(\lambda \mathbb{1}_{A_{+}}-\mathbb{1}_{A_{-}}\right)|Q|^{\frac{1}{p}} \cdot\left(\lambda^{\left(1-p^{\prime}\right)(p-1)} \phi+\psi\right) \\
& =|Q|^{\frac{1}{p}} \cdot(\phi-\psi) .
\end{aligned}
$$

Applying $\mathbf{R}$ then gives $\mathbf{R}\left(Q_{\lambda}|u|^{p-2} u\right)=\mathbf{R}\left(|Q|^{\frac{1}{p}}(\phi-\psi)\right)=u$. Hence $u$ solves (2.1).

So we conclude that it remains to find nontrivial critical points of the functionals $J_{\lambda}$ for as many $\lambda>0$ as possible. This will be achieved with the Mountain Pass Theorem for families of $\mathcal{C}^{1}$-functionals by Jeanjean and Toland [6].

\section{Saddle-point reduction}

In this section we perform the saddle-point reduction of $J_{\lambda}$ with respect to the $\psi$-variable. To this end, we prove that for any fixed $\phi \in L^{p^{\prime}}\left(A_{+}\right)$the functional $\psi \mapsto J_{\lambda}(\phi, \psi)$ attains its maximum at some uniquely defined function in $L^{p^{\prime}}\left(A_{-}\right)$that we will call $Z(\phi)$ in the following. We shall see that the positivity assumption $\int \psi \mathcal{K} \psi d x \geq 0$ for all $\psi \in L^{p^{\prime}}\left(A_{-}\right)$ 
ensures that the functional $\psi \mapsto J_{\lambda}(\phi, \psi)$ is strictly concave so that the global maximization with respect to $\psi$ is the only reasonable approach to perform a saddle point reduction. We introduce the reduced functional $\tilde{J}_{\lambda}: L^{p^{\prime}}\left(A_{+}\right) \rightarrow \mathbb{R}$ via

$$
\tilde{J}_{\lambda}(\phi):=\sup _{\psi \in L^{p^{\prime}}\left(A_{-}\right)} J_{\lambda}(\phi, \psi) \text {. }
$$

Proposition 3.1 Assume that $\mathcal{K}: L^{p^{\prime}}\left(\mathbb{R}^{N}\right) \rightarrow L^{p}\left(\mathbb{R}^{N}\right)$ is compact and that $\int_{A_{-}} \psi \mathcal{K} \psi d x \geq 0$ for all $\psi \in L^{p^{\prime}}\left(A_{-}\right)$. Then for every $\phi \in L^{p^{\prime}}\left(A_{+}\right)$there exists a unique $Z(\phi) \in L^{p^{\prime}}\left(A_{-}\right)$ such that for all $\lambda>0$ we have

$$
\tilde{J}_{\lambda}(\phi)=J_{\lambda}(\phi, Z(\phi))
$$

Moreover:

(i) For any $\phi \in L^{p^{\prime}}\left(A_{+}\right)$the corresponding maximizer $Z(\phi)$ satisfies

$$
\|Z(\phi)\|_{p^{\prime}} \leq\left(p^{\prime} \beta\|\phi\|_{p^{\prime}}\right)^{\frac{1}{p^{\prime}-1}}
$$

where $\beta$ is defined in (1.8).

(ii) The map $Z: L^{p^{\prime}}\left(A_{+}\right) \rightarrow L^{p^{\prime}}\left(A_{-}\right)$is continuous.

(iii) The reduced functional $\tilde{J}_{\lambda}: L^{p^{\prime}}\left(A_{+}\right) \rightarrow \mathbb{R}$ is of class $\mathcal{C}^{1}$ with derivative

$$
\tilde{J}_{\lambda}^{\prime}[h]=\partial_{1} J_{\lambda}(\phi, Z(\phi))[h] .
$$

Proof We first establish the existence of a maximizer. So fix $\phi \in L^{p^{\prime}}\left(A_{+}\right)$and consider a maximizing sequence $\left(\psi_{n}\right)_{n} \subset L^{p^{\prime}}\left(A_{-}\right)$. Using $J_{\lambda}(\phi, 0) \leq \sup _{\lambda} J_{\lambda}(\phi, \psi)=$ $J_{\lambda}\left(\phi, \psi_{n}\right)+o(1)$ as $n \rightarrow \infty$ we obtain

$$
o(1) \leq-\frac{1}{p^{\prime}}\left\|\psi_{n}\right\|_{p^{\prime}}^{p^{\prime}}+\int_{\mathbb{R}^{N}} \phi \mathcal{K} \psi_{n}-\frac{1}{2} \int_{\mathbb{R}^{N}} \psi_{n} \mathcal{K} \psi_{n} \leq-\frac{1}{p^{\prime}}\left\|\psi_{n}\right\|_{p^{\prime}}^{p^{\prime}}+\beta\|\phi\|_{p^{\prime}}\left\|\psi_{n}\right\|_{p^{\prime}} \quad(n \rightarrow \infty) .
$$

Here we used the nonnegativity assumption on $\mathcal{K}$ as well as (1.8). Hence,

$$
\left\|\psi_{n}\right\|_{p^{\prime}} \leq\left(p^{\prime} \beta\|\phi\|\right)^{\frac{1}{p^{\prime}-1}}+o(1) \quad(n \rightarrow \infty)
$$

so $\left(\psi_{n}\right)_{n}$ is bounded. Passing to a subsequence we find $\psi^{*} \in L^{p^{\prime}}\left(A_{-}\right)$such that $\psi_{n} \rightarrow \psi^{*}$ in $L^{p^{\prime}}\left(A_{-}\right)$as $n \rightarrow \infty$. Using the compactness of $\mathcal{K}$ and the weak lower semicontinuity of the norm we find

$$
\begin{aligned}
& \sup _{\psi \in L^{p^{\prime}}\left(A_{-}\right)} J_{\lambda}(\phi, \psi) \\
& =\frac{\lambda^{1-p^{\prime}}}{p^{\prime}}\|\phi\|_{p^{\prime}}^{p^{\prime}}-\frac{1}{p^{\prime}}\left\|\psi_{n}\right\|_{p^{\prime}}^{p^{\prime}}-\frac{1}{2} \int_{\mathbb{R}^{N}} \phi \mathcal{K} \phi d x+\int_{\mathbb{R}^{N}} \phi \mathcal{K} \psi_{n} d x-\frac{1}{2} \int_{\mathbb{R}^{N}} \psi_{n} \mathcal{K} \psi_{n} d x+o(1) \\
& =\frac{\lambda^{1-p^{\prime}}}{p^{\prime}}\|\phi\|_{p^{\prime}}^{p^{\prime}}-\frac{1}{p^{\prime}}\left\|\psi_{n}\right\|_{p^{\prime}}^{p^{\prime}}-\frac{1}{2} \int_{\mathbb{R}^{N}} \phi \mathcal{K} \phi d x+\int_{\mathbb{R}^{N}} \phi \mathcal{K} \psi^{*} d x-\frac{1}{2} \int_{\mathbb{R}^{N}} \psi^{*} \mathcal{K} \psi^{*} d x+o(1) \\
& \leq \frac{\lambda^{1-p^{\prime}}}{p^{\prime}}\|\phi\|_{p^{\prime}}^{p^{\prime}}-\frac{1}{p^{\prime}}\left\|\psi^{*}\right\|_{p^{\prime}}^{p^{\prime}}-\frac{1}{2} \int_{\mathbb{R}^{N}} \phi \mathcal{K} \phi d x+\int_{\mathbb{R}^{N}} \phi \mathcal{K} \psi^{*} d x-\frac{1}{2} \int_{\mathbb{R}^{N}} \psi^{*} \mathcal{K} \psi^{*} d x+o(1) \\
& =J_{\lambda}\left(\phi, \psi^{*}\right)+o(1) .
\end{aligned}
$$


Hence the supremum is attained at $\psi^{*}$. Since equality must hold in the above estimate we conclude $\left\|\psi_{n}\right\|_{p^{\prime}} \rightarrow\left\|\psi^{*}\right\|_{p^{\prime}}$, whence $\psi_{n} \rightarrow \psi^{*}$ in $L^{p^{\prime}}\left(A_{-}\right)$as $n \rightarrow \infty$. This shows the existence of a maximizer satisfying the estimate stated in (i). So (i) is proved once we have established the uniqueness of the maximizer.

To this end assume that $\psi^{*}, \psi^{\dagger} \in L^{p^{\prime}}\left(A_{-}\right)$are maximizers. Then we have

$$
\begin{aligned}
0 \leq & \frac{1}{2} J_{\lambda}\left(\phi, \psi^{*}\right)+\frac{1}{2} J_{\lambda}\left(\phi, \psi^{\dagger}\right)-J_{\lambda}\left(\phi, \frac{1}{2}\left(\psi^{*}+\psi^{\dagger}\right)\right) \\
= & \frac{1}{p^{\prime}}\left(\left\|\frac{\psi^{*}+\psi^{\dagger}}{2}\right\|_{p^{\prime}}^{p^{\prime}}-\frac{1}{2}\left\|\psi^{*}\right\|_{p^{\prime}}^{p^{\prime}}-\frac{1}{2}\left\|\psi^{\dagger}\right\|_{p^{\prime}}^{p^{\prime}}\right) \\
& +\frac{1}{2}\left(\int_{\mathbb{R}^{N}} \frac{\psi^{*}+\psi^{\dagger}}{2} \mathcal{K}\left[\frac{\psi^{*}+\psi^{\dagger}}{2}\right] \mathrm{d} x-\frac{1}{2} \int_{\mathbb{R}^{N}} \psi^{*} \mathcal{K} \psi^{*} \mathrm{~d} x-\frac{1}{2} \int_{\mathbb{R}^{N}} \psi^{\dagger} \mathcal{K} \psi^{\dagger} \mathrm{d} x\right) \\
= & \frac{1}{p^{\prime}}\left(\left\|\frac{\psi^{*}+\psi^{\dagger}}{2}\right\|_{p^{\prime}}^{p^{\prime}}-\frac{1}{2}\left\|\psi^{*}\right\|_{p^{\prime}}^{p^{\prime}}-\frac{1}{2}\left\|\psi^{\dagger}\right\|_{p^{\prime}}^{p^{\prime}}\right)-\frac{1}{8} \int_{\mathbb{R}^{N}}\left(\psi^{*}-\psi^{\dagger}\right) \mathcal{K}\left[\psi^{*}-\psi^{\dagger}\right] \mathrm{d} x \\
\leq & \frac{1}{p^{\prime}}\left(\left\|\frac{\psi^{*}+\psi^{\dagger}}{2}\right\|_{p^{\prime}}^{p^{\prime}}-\frac{1}{2}\left\|\psi^{*}\right\|_{p^{\prime}}^{p^{\prime}}-\frac{1}{2}\left\|\psi^{\dagger}\right\|_{p^{\prime}}^{p^{\prime}}\right) \\
\leq & 0,
\end{aligned}
$$

where we have used the nonnegativity condition in the second last step and the convexity of $z \mapsto|z|^{p^{\prime}}$ in the last step. So we have equality in each estimate and conclude $\psi^{*}=\psi^{\dagger}$. Note that the maximizer does not depend on $\lambda$ since the only $\lambda$-dependent term in $J_{\lambda}(\phi, \psi)$ is $\frac{\lambda^{1-p^{\prime}}}{p^{\prime}}\|\phi\|_{p^{\prime}}^{p^{\prime}}$, which is independent of $\psi$.

We now prove (ii), i.e., the continuity of the map $Z$ : Assume $\phi_{n} \rightarrow \phi$ in $L^{p^{\prime}}\left(A_{+}\right)$and let $\left(\psi_{n}\right)_{n}:=\left(Z\left(\phi_{n}\right)\right)_{n} \subset L^{p^{\prime}}\left(A_{-}\right)$be the associated maximizers. By (3.3), the sequence $\left(\psi_{n}\right)_{n}$ is bounded and after passing to a subsequence we may assume $\psi_{n} \rightarrow \psi_{0}$ in $L^{p^{\prime}}\left(A_{-}\right)$ as $n \rightarrow \infty$. Arguing as above we deduce

$$
\limsup _{n \rightarrow \infty} \tilde{J}_{\lambda}\left(\phi_{n}\right)=\limsup _{n \rightarrow \infty} J_{\lambda}\left(\phi_{n}, \psi_{n}\right)=\limsup _{n \rightarrow \infty} J_{\lambda}\left(\phi, \psi_{n}\right) \leq J_{\lambda}\left(\phi, \psi_{0}\right) \leq \tilde{J}_{\lambda}(\phi)
$$

using weak lower semicontinuity and $\liminf _{n \rightarrow \infty}\left\|\psi_{n}\right\|_{p^{\prime}} \geq\left\|\psi_{0}\right\|_{p^{\prime}}$. On the other hand, with the special choice $\psi=Z(\phi)$ we obtain

$$
\liminf _{n \rightarrow \infty} \tilde{J}_{\lambda}\left(\phi_{n}\right) \geq J_{\lambda}(\phi, \psi)=J_{\lambda}(\phi, Z(\phi))=\tilde{J}_{\lambda}(\phi)
$$

Combining both estimates gives $\left\|\psi_{n}\right\|_{p^{\prime}} \rightarrow\left\|\psi_{0}\right\|_{p^{\prime}}$ as well as $\tilde{J}_{\lambda}\left(\phi_{n}\right) \rightarrow \tilde{J}_{\lambda}(\phi)$ as $n \rightarrow \infty$. Thus we have equality in (3.4), (3.5). Since maximizers are unique, we obtain $\psi_{0}=Z(\phi)$ and in particular $Z\left(\phi_{n}\right)=\psi_{n} \rightarrow \psi_{0}=Z\left(\phi_{0}\right)$ in $L^{p^{\prime}}\left(A_{-}\right)$as $n \rightarrow \infty$. 
We are left to prove (iii). Let $h \in L^{p^{\prime}}\left(A_{+}\right)$be arbitrary. We can estimate the difference quotients as follows:

$$
\begin{aligned}
\liminf _{\tau \rightarrow 0} \frac{\tilde{J}_{\lambda}(\phi+\tau h)-\tilde{J}_{\lambda}(\phi)}{\tau} & \geq \liminf _{\tau \rightarrow 0} \frac{J_{\lambda}(\phi+\tau h, Z(\phi))-J_{\lambda}(\phi, Z(\phi))}{\tau} \\
& =\liminf _{\tau \rightarrow 0} \int_{0}^{1} \partial_{1} J_{\lambda}(\phi+\tau \sigma h, Z(\phi))[h] \mathrm{d} \sigma \\
& =\partial_{1} J_{\lambda}(\phi, Z(\phi))[h], \\
\limsup _{\tau \rightarrow 0} \frac{\tilde{J}_{\lambda}(\phi+\tau h)-\tilde{J}_{\lambda}(\phi)}{\tau} & \leq \limsup _{\tau \rightarrow 0} \frac{J_{\lambda}(\phi+\tau h, Z(\phi+\tau h))-J_{\lambda}(\tilde{\phi}, Z(\phi+\tau h))}{\tau} \\
& =\limsup _{\tau \rightarrow 0} \int_{0}^{1} \partial_{1} J_{\lambda}\left(\phi^{*}+\tau \sigma h, Z(\phi+\tau h)\right)[h] \mathrm{d} \sigma \\
& =\partial_{1} J_{\lambda}(\phi, Z(\phi))[h] .
\end{aligned}
$$

Here we used that $Z$ is continuous and that $\partial_{1} J_{\lambda}$ is continuous, see [10, Proposition 9] for a similar computation. We conclude that $\tilde{J}_{\lambda}$ is Gâteaux-differentiable with continuous derivative $\phi \mapsto \partial_{1} J_{\lambda}(\phi, Z(\phi))[\cdot]$, see Proposition 3.1 (iii). Hence, the reduced functional $\tilde{J}_{\lambda}$ is continuously (Fréchet-)differentiable with

$$
\tilde{J}_{\lambda}^{\prime}(\phi)[h]=\partial_{1} J_{\lambda}(\phi, Z(\phi))[h] \quad \forall h \in L^{p^{\prime}}\left(A_{+}\right)
$$

as claimed.

Notice that the condition $\int_{\mathbb{R}^{N}} \psi \mathcal{K} \psi \mathrm{d} x \geq 0$ is also necessary for the existence of a global maximizer of $\psi \mapsto J_{\lambda}(\phi, \psi)$ because otherwise this functional is unbounded from above.

\section{Palais-Smale sequences for the reduced functional}

In view of the results of the previous sections, we obtain a solution to our problem by proving the existence of a nontrivial critical point of the reduced functional $\tilde{J}_{\lambda}: L^{p^{\prime}}\left(A_{+}\right) \rightarrow \mathbb{R}$ introduced in (3.1). This will be done via Mountain-pass techniques for monotone families of functionals originating from the work of Jeanjean and Toland [6].

Definition 4.1 Let $X$ be a Banach space, $M \subset \mathbb{R}$ a compact interval. Then the family $\left(I_{\nu}\right)_{\nu \in M}$ of $\mathcal{C}^{1}$-functionals on $X$ is said to have the Mountain Pass Geometry if there exist $v_{1}, v_{2} \in X$ such that for all $v \in M$ it holds

$$
c_{v}:=\inf _{\gamma \in \Gamma} \sup _{t \in[0,1]} I_{v}(\gamma(t))>\max \left\{I_{v}\left(v_{1}\right), I_{v}\left(v_{2}\right)\right\}
$$

where $\Gamma:=\left\{\gamma \in C([0,1], X): \gamma(0)=v_{1}, \gamma(1)=v_{2}\right\}$.

Theorem 4.2 (Jeanjean, Toland) [6, Theorem 2.1] Assume that $X$ is a Banach space, $M \subset \mathbb{R}$ a compact interval and $\left(I_{\nu}\right)_{v \in M}$ a family of $\mathcal{C}^{1}$-functionals on $X$ having the Mountain Pass Geometry. Assume further that $\left(I_{v}\right)_{v \in M}$ has the following property:

For every sequence $\left(v_{n}, \phi_{n}\right) \in M \times X$ with $v_{n} \nearrow v_{*} \in M$ and with

$$
-I_{v_{*}}\left(\phi_{n}\right), \quad I_{v_{n}}\left(\phi_{n}\right), \quad \frac{I_{v_{n}}\left(\phi_{n}\right)-I_{v_{*}}\left(\phi_{n}\right)}{v_{*}-v_{n}} \text { bounded from above, }
$$

the sequence $\left(\phi_{n}\right)$ is bounded itself, and $\lim \sup \left(I_{\nu_{*}}\left(\phi_{n}\right)-I_{v_{n}}\left(\phi_{n}\right)\right) \leq 0$. 
Then for almost all $v \in M$ there is a bounded Palais-Smale sequence (BPS) for $I_{v}$ at the level $c_{v}$.

We shall apply this result to $X=L^{p^{\prime}}\left(A_{+}\right)$and the family of $\mathcal{C}^{1}$-functionals $I_{\lambda}:=\tilde{J}_{\lambda}$ : $X \rightarrow \mathbb{R}$. We first verify the Mountain Pass Geometry for parameters $\lambda \in\left(\lambda_{0}, \infty\right)$ where $\lambda_{0}=\left(2 \beta \alpha^{-1}\right)^{p}$. Let us recall that $\alpha, \beta$ were defined as

$$
\alpha:=\max _{\substack{\|\phi\|_{p^{\prime}}=1, \operatorname{supp}(\phi) \subset A_{+}}} \int_{\mathbb{R}^{N}} \phi \mathcal{K} \phi d x, \quad \beta:=\max _{\begin{array}{c}
\|\phi\|_{p^{\prime}}=\|\psi\|_{p^{\prime}}=1, \\
\operatorname{supp}(\phi) \subset A_{+}, \operatorname{supp}(\psi) \subset A_{-}
\end{array}} \int_{\mathbb{R}^{N}} \phi \mathcal{K} \psi d x .
$$

Proposition 4.3 Let $\mathcal{K}: L^{p^{\prime}}\left(\mathbb{R}^{N}\right) \rightarrow L^{p}\left(\mathbb{R}^{N}\right)$ be compact and assume $\int_{\mathbb{R}^{N}} \psi \mathcal{K} \psi d x \geq 0$ for all $\psi \in L^{p^{\prime}}\left(A_{-}\right)$. Then, for any given compact subinterval $M \subset\left(\lambda_{0}, \infty\right)$, the family of functionals $\left(\tilde{J}_{\lambda}\right)_{\lambda \in M}$ has the Mountain Pass Geometry according to Definition 4.1.

Proof For $\lambda \in M$ we define $r_{\lambda}:=\left(\lambda^{p^{\prime}-1} \alpha\right)^{1 /\left(p^{\prime}-2\right)}$. Then we have

$$
\begin{aligned}
\inf _{\|\phi\|_{p^{\prime}}=r_{\lambda}} \tilde{J}_{\lambda}(\phi) & =\inf _{\|\phi\|_{p^{\prime}}=r_{\lambda}} \sup _{\psi \in L^{p^{\prime}}\left(A_{-}\right)} J_{\lambda}(\phi, \psi) \geq \inf _{\|\phi\|_{p^{\prime}}=r_{\lambda}} J_{\lambda}(\phi, 0) \\
& =\inf _{\|\phi\|_{p^{\prime}}=r_{\lambda}} \frac{\lambda^{1-p^{\prime}}}{p^{\prime}}\|\phi\|_{p^{\prime}}^{p^{\prime}}-\frac{1}{2} \int_{\mathbb{R}^{N}} \phi \mathcal{K} \phi \mathrm{d} x \\
& =\frac{\lambda^{1-p^{\prime}}}{p^{\prime}} r_{\lambda}^{p^{\prime}}-\frac{\alpha}{2} r_{\lambda}^{2}=\alpha\left(\frac{1}{p^{\prime}}-\frac{1}{2}\right)\left(\lambda^{p^{\prime}-1} \alpha\right)^{\frac{2}{p^{\prime}-2}} \\
& >0 .
\end{aligned}
$$

On the other hand, we have

$$
\tilde{J}_{\lambda}(0)=\sup _{\psi \in L^{p^{\prime}}\left(A_{-}\right)} J_{\lambda}(0, \psi)=\sup _{\psi \in L^{p^{\prime}\left(A_{-}\right)}}\left[-\frac{1}{p^{\prime}}\|\psi\|_{p^{\prime}}^{p^{\prime}}-\frac{1}{2} \int_{\mathbb{R}^{N}} \psi \mathcal{K} \psi \mathrm{d} x\right]=0 .
$$

According to Definition 4.1 it therefore remains to find some $\phi^{*} \in L^{p^{\prime}}\left(A_{+}\right)$with $\left\|\phi^{*}\right\|_{p^{\prime}} \geq$ $r_{\lambda_{0}}$ such that $\tilde{J}_{\lambda_{0}}\left(\phi^{*}\right) \leq 0$ holds. Notice that in this case we actually have $\tilde{J}_{\lambda}\left(\phi^{*}\right)<\tilde{J}_{\lambda_{0}}\left(\phi^{*}\right) \leq$ 0 for all $\lambda \in M \subset\left(\lambda_{0}, \infty\right)$. To achieve this we estimate $\tilde{J}_{\lambda_{0}}$ from above as follows

$$
\begin{aligned}
\tilde{J}_{\lambda_{0}}(\phi) & =J_{\lambda_{0}}(\phi, 0)-\frac{1}{p^{\prime}}\|Z(\phi)\|_{p^{\prime}}^{p^{\prime}}+\int_{\mathbb{R}^{N}} \phi \mathcal{K}(Z(\phi))-\frac{1}{2} \int_{\mathbb{R}^{N}} Z(\phi) \mathcal{K}(Z(\phi)) \\
& \leq J_{\lambda_{0}}(\phi, 0)-\frac{1}{p^{\prime}}\|Z(\phi)\|_{p^{\prime}}^{p^{\prime}}+\beta\|\phi\|_{p^{\prime}}\|Z(\phi)\|_{p^{\prime}} \\
& \leq J_{\lambda_{0}}(\phi, 0)+\frac{\beta^{p}}{p}\|\phi\|_{p^{\prime}}^{p}
\end{aligned}
$$

where we have used that $\max _{c \geq 0}\left(-\frac{c^{p^{\prime}}}{p^{\prime}}+\beta\|\phi\|_{p^{\prime}} c\right)=\frac{\beta^{p}}{p}\|\phi\|_{p^{\prime}}^{p}$. We choose $\phi^{*}=R \phi_{0}$ where the function $\phi_{0} \in L^{p^{\prime}}\left(A_{+}\right),\left\|\phi_{0}\right\|_{p^{\prime}}=1$ attains the maximum $\alpha=\int_{\mathbb{R}^{N}} \phi_{0} \mathcal{K} \phi_{0} \mathrm{~d} x>0$. Then the choice $R:=\left(\frac{1}{2} \alpha \beta^{-p}\right)^{1 /(p-2)}$ yields after some computations $\left(\right.$ recall $\left.\lambda_{0}=\left(2 \beta \alpha^{-1}\right)^{p}\right)$ $\left\|\phi^{*}\right\|_{p^{\prime}}=R=\left(\frac{1}{2} \alpha \beta^{-p}\right)^{\frac{1}{p-2}} \geq\left(\lambda_{0}^{p^{\prime}-1} \alpha\right)^{\frac{1}{p^{\prime}-2}}>\left(\lambda^{p^{\prime}-1} \alpha\right)^{\frac{1}{p^{\prime}-2}}=r_{\lambda} \quad$ for all $\lambda \in M$. 
Using again the explicit formulas for $R, \lambda_{0}$ we find

$$
\begin{aligned}
\tilde{J}_{\lambda_{0}}\left(R \phi_{0}\right) & \leq J_{\lambda_{0}}\left(R \phi_{0}, 0\right)+\frac{\beta^{p} R^{p}}{p} \\
& =\frac{\lambda_{0}^{1-p^{\prime}}}{p^{\prime}} R^{p^{\prime}}-\frac{\alpha}{2} R^{2}+\frac{\beta^{p}}{p} R^{p} \\
& =\frac{R^{p^{\prime}}}{p^{\prime}} \cdot\left(\lambda_{0}^{1-p^{\prime}}-\frac{p \alpha}{2(p-1)} R^{2-p^{\prime}}+\frac{\beta^{p}}{p-1} R^{p-p^{\prime}}\right) \\
& =\frac{R^{p^{\prime}}}{p^{\prime}} \cdot\left(\lambda_{0}^{1-p^{\prime}}-\frac{1}{2} \alpha R^{2-p^{\prime}}\right) \\
& =\frac{R^{p^{\prime}}}{p^{\prime}} \cdot\left(\left(2 \beta \alpha^{-1}\right)^{-\frac{p}{p-1}}-\frac{1}{2} \alpha \cdot\left(\frac{1}{2} \alpha \beta^{-p}\right)^{\frac{1}{p-1}}\right) \\
& =0
\end{aligned}
$$

and thus the claim holds with $v_{1}=0$ and $v_{2}=\phi^{*}=R \phi_{0}$.

Having established the Mountain Pass Geometry of our functionals we now verify the condition $(\mathrm{H})$ in order to use Theorem 4.2

Proposition 4.4 For any compact subinterval $M \subset\left(\lambda_{0}, \infty\right)$ the family of $\mathcal{C}^{1}$-functionals $\left(\tilde{J}_{\lambda}\right)_{\lambda \in M}$ satisfies the condition $(\mathrm{H})$.

Proof Consider a sequence $\left(\phi_{n}, \lambda_{n}\right) \in L^{p^{\prime}}\left(A_{+}\right) \times M$ with $\lambda_{n} \nearrow \lambda_{*}$ and

$$
-\tilde{J}_{\lambda_{*}}\left(\phi_{n}\right) \leq C, \quad \tilde{J}_{\lambda_{n}}\left(\phi_{n}\right) \leq C, \quad \frac{\tilde{J}_{\lambda_{n}}\left(\phi_{n}\right)-\tilde{J}_{\lambda_{*}}\left(\phi_{n}\right)}{\lambda_{*}-\lambda_{n}} \leq C
$$

for all $n \in \mathbb{N}$. Then we have

$$
\begin{aligned}
C \geq \frac{\tilde{J}_{\lambda_{n}}\left(\phi_{n}\right)-\tilde{J}_{\lambda^{*}}\left(\phi_{n}\right)}{\lambda_{*}-\lambda_{n}} & =\frac{\lambda_{n}^{1-p^{\prime}}-\lambda_{*}^{1-p^{\prime}}}{\lambda_{*}-\lambda_{n}}\left\|\phi_{n}\right\|_{p^{\prime}}^{p^{\prime}} \\
& =\left(\left(p^{\prime}-1\right) \lambda_{*}^{-p^{\prime}}+o(1)\right)\left\|\phi_{n}\right\|_{p^{\prime}}^{p^{\prime}} \quad(n \rightarrow \infty)
\end{aligned}
$$

So we conclude that $\left(\phi_{n}\right)$ is bounded. Furthermore, $\lambda_{n} \rightarrow \lambda^{*}>0$ gives

$$
\limsup _{n \rightarrow \infty}\left(I_{\lambda_{*}}\left(\phi_{n}\right)-I_{\lambda_{n}}\left(\phi_{n}\right)\right)=\limsup _{n \rightarrow \infty}\left(\lambda_{n}^{1-p^{\prime}}-\lambda_{*}^{1-p^{\prime}}\right)\left\|\phi_{n}\right\|_{p^{\prime}}^{p^{\prime}}=0,
$$

which is all we had to show.

We thus conclude that Theorem 4.2 applies in our context and yields BPS sequences for $\tilde{J}_{\lambda}$ at the corresponding Mountain pass levels $c_{\lambda}$ for almost all $\lambda \in\left(\lambda_{0}, \infty\right)$. From the existence of BPS sequences we deduce rather easily the existence of critical points at the corresponding Mountain Pass level.

Proposition 4.5 Let $\mathcal{K}: L^{p^{\prime}}\left(\mathbb{R}^{N}\right) \rightarrow L^{p}\left(\mathbb{R}^{N}\right)$ be compact and assume $\int_{\mathbb{R}^{N}} \psi \mathcal{K} \psi d x \geq 0$ for all $\psi \in L^{p^{\prime}}\left(A_{-}\right)$. Then for all $\lambda \in\left(\lambda_{0}, \infty\right)$ every BPS sequence of $\tilde{J}_{\lambda}$ at its Mountain Pass level $c_{\lambda}$ converges to a critical point of $\tilde{J}_{\lambda}$ at the level $c_{\lambda}$. 
Proof Let $\left(\phi_{j}\right)_{j}$ in $L^{p^{\prime}}\left(A_{+}\right)$be a BPS sequence for $\tilde{J}_{\lambda}$, i.e., $\tilde{J}_{\lambda}\left(\phi_{j}\right) \rightarrow c>0$ and $\tilde{J}_{\lambda}^{\prime}\left(\phi_{j}\right) \rightarrow 0$. We may thus assume w.l.o.g. $\phi_{j} \rightarrow \phi^{*}$. Moreover, Proposition 3.1 (i) implies the boundedness of $\left(\psi_{j}\right)_{j}:=\left(Z\left(\phi_{j}\right)\right)_{j}$ and hence w.l.o.g. also weak convergence. For all $h \in L^{p^{\prime}}\left(A_{+}\right)$we then have, in view of the formula for $\tilde{J}_{\lambda}^{\prime}$ from Proposition 3.1 (iii),

$$
\begin{aligned}
& \left.\left|\int_{\mathbb{R}^{N}}\right| \phi_{j}\right|^{p^{\prime}-2} \phi_{j} h-\left|\phi_{k}\right|^{p^{\prime}-2} \phi_{k} h \mathrm{~d} x \mid \\
& \quad=\left|\tilde{J}_{\lambda}^{\prime}\left(\phi_{j}\right) h-\tilde{J}_{\lambda}^{\prime}\left(\phi_{k}\right) h+\int_{\mathbb{R}^{N}} h \mathcal{K}\left[\phi_{j}-\phi_{k}\right] \mathrm{d} x-\int_{\mathbb{R}^{N}} h \mathcal{K}\left[\psi_{j}-\psi_{k}\right] \mathrm{d} x\right| \\
& \quad \leq\|h\|_{p^{\prime}} \cdot\left[\left\|\tilde{J}_{\lambda}^{\prime}\left(\phi_{j}\right)\right\|+\left\|\tilde{J}_{\lambda}^{\prime}\left(\phi_{k}\right)\right\|+\left\|\mathcal{K}\left[\phi_{j}-\phi_{k}\right]\right\|_{p}+\left\|\mathcal{K}\left[\psi_{j}-\psi_{k}\right]\right\|_{p}\right] \\
& \quad=\|h\|_{p^{\prime}} \cdot o(1) \quad(j, k \rightarrow \infty) .
\end{aligned}
$$

We infer that $\left(\left|\phi_{j}\right|^{p^{\prime}-2} \phi_{j}\right)_{j}$ converges strongly in $L^{p}\left(A_{+}\right)$. By uniqueness of weak limits, we infer $\left|\phi_{j}\right|^{p^{\prime}-2} \phi_{j} \rightarrow\left|\phi^{*}\right|^{p^{\prime}-2} \phi^{*}$ strongly in $L^{p}\left(A_{+}\right)$and hence in particular $\left\|\phi_{j}\right\|_{p^{\prime}} \rightarrow$ $\left\|\phi^{*}\right\|_{p^{\prime}}$. This finally implies $\phi_{j} \rightarrow \phi^{*}$ strongly in $L^{p^{\prime}}\left(A_{+}\right)$. A standard computation finally shows $\tilde{J}_{\lambda}\left(\phi^{*}\right)=c_{\lambda}$ as well as $\tilde{J}_{\lambda}^{\prime}\left(\phi^{*}\right)=0$.

\section{Proof of Theorem 1.1 and Corollary 1.2}

We finally combine all auxiliary results to prove Theorem 1.1 .

Proof of Theorem 1.1 From Proposition 2.1 and Proposition 3.1 we infer that for almost all $\lambda \in\left(\lambda_{0}, \infty\right)$ a nontrivial solution $u \in L^{p}\left(\mathbb{R}^{N}\right)$ of the nonlinear Helmholtz equation (1.10) is found once we have proved the existence of nontrivial critical points of the reduced functional $\tilde{J}_{\lambda}$ for almost all $\lambda \in M$ where $M$ is an arbitrary compact subinterval of $\left(\lambda_{0}, \infty\right)$. From Proposition 4.3 we infer that the family $\left(\tilde{J}_{\lambda}\right)_{\lambda \in M}$ has the Mountain Pass Geometry. Moreover, by Proposition 4.4, condition (H) holds. So Theorem 4.2 yields for almost all $\lambda \in M$ a BPS sequence for $\tilde{J}_{\lambda}$ at the corresponding Mountain Pass level. By Proposition 4.5 each of these BPS sequences converges to a critical point $\phi_{\lambda}$ of $\tilde{J}_{\lambda}$ at the Mountain Pass level. Since this critical point is necessarily nontrivial, we have thus obtained the desired claim for $L^{p}\left(\mathbb{R}^{N}\right)$ solutions of (1.10). From [4, Lemma 4.3] we infer that each of these solutions belongs to $W^{2, q}\left(\mathbb{R}^{N}\right) \cap \mathcal{C}^{1, \alpha}\left(\mathbb{R}^{N}\right)$ for all $p \leq q<\infty$ and $\alpha \in(0,1)$. Arguing as in Step 3 and Step 4 $[8, \mathrm{p} .13]$ one even obtains that these solutions belong to $W^{2, q}\left(\mathbb{R}^{N}\right)$ for all $q \in\left(\frac{2 N}{N-1}, p\right)$. In particular, these solutions are strong solutions of (1.10), which finishes the proof.

Proof of Collorary 1.2 In order to apply Theorem 1.1 we show that (1.11) implies (1.9). In the special case $k=1$ the compactness of $\mathcal{K}$ was shown in Lemma 4.2 in [4]. So the general case follows by rescaling. It therefore remains to show that $\delta:=\operatorname{diam}\left(A_{-}\right) \leq k^{-1} y_{\frac{N-2}{2}}$ implies $\int \psi \mathcal{K} \psi d x \geq 0$ for all $\psi \in L^{p^{\prime}}\left(A_{-}\right)$. Due to (2.2), $\mathcal{K}=|Q|^{1 / p} \mathbf{R}\left(|Q|^{1 / p}\right.$.) and $\mathbb{R}^{N}$ $Q \in L^{\infty}\left(\mathbb{R}^{N}\right)$ it suffices to prove

$$
\int_{\mathbb{R}^{N}} \psi \mathbf{R} \psi d x \geq 0, \quad \text { for all } \psi \in \mathcal{S}\left(A_{-}\right) .
$$


Using that $x, y \in A_{-}$implies $x-y \in B_{\delta}$ we infer from Corollary 5.4 in [2]

$$
\int_{\mathbb{R}^{N}} \psi \mathbf{R} \psi d x=\int_{\mathbb{R}^{N}} \psi\left[\mathbb{1}_{B_{\delta}} \Psi * \psi\right](x) d x \geq 0,
$$

which proves (5.1) and hence the Corollary.

Acknowledgements The authors thank Tobias Weth for helpful suggestions and stimulating discussions. The first two authors are funded by the Deutsche Forschungsgemeinschaft (DFG, German Research Foundation)Project-ID 258734477-SFB 1173.

Funding Open Access funding enabled and organized by Projekt DEAL.

Open Access This article is licensed under a Creative Commons Attribution 4.0 International License, which permits use, sharing, adaptation, distribution and reproduction in any medium or format, as long as you give appropriate credit to the original author(s) and the source, provide a link to the Creative Commons licence, and indicate if changes were made. The images or other third party material in this article are included in the article's Creative Commons licence, unless indicated otherwise in a credit line to the material. If material is not included in the article's Creative Commons licence and your intended use is not permitted by statutory regulation or exceeds the permitted use, you will need to obtain permission directly from the copyright holder. To view a copy of this licence, visit http://creativecommons.org/licenses/by/4.0/.

\section{References}

1. Abramowitz, M., Stegun, I. A.: Handbook of Mathematical Functions with Formulas, Graphs, and Mathematical Tables, p. 1046. National Bureau of Standards Applied Mathematics Series 55, Washington, D.C., (1964)

2. Chen, H., Evéquoz, G., Weth, T.: Complex solutions and stationary scattering for the nonlinear Helmholtz equation. arXiv:1911.09557 (2019)

3. Evéquoz, G., Weth, T.: Real solutions to the nonlinear Helmholtz equation with local nonlinearity. Arch. Ration. Mech. Anal. 211(2), 359-388 (2014)

4. Evéquoz, G., Weth, T.: Dual variational methods and nonvanishing for the nonlinear Helmholtz equation. Adv. Math. 280, 690-728 (2015)

5. Evéquoz, G., Yeşil, T.: Dual ground state solutions for the critical nonlinear Helmholtz equation. Proc. R. Soc. Edinb. Sect. A: Math. 150(3), 1155-1186 (2020)

6. Jeanjean, L., Toland, J.F.: Bounded Palais-Smale mountain-pass sequences. C. R. Acad. Sci. Paris Sér. I Math. 327(1), 23-28 (1998)

7. Kenig, C.E., Ruiz, A., Sogge, C.D., et al.: Uniform Sobolev inequalities and unique continuation for second order constant coefficient differential operators. Duke Math. J. 55(2), 329-347 (1987)

8. Mandel, R.: Uncountably many solutions for nonlinear Helmholtz and curl-curl equations. Adv. Nonlinear Stud. 19(3), 569-593 (2019)

9. Mandel, R., Montefusco, E., Pellacci, B.: Oscillating solutions for nonlinear Helmholtz equations. Z. Angew. Math. Phys. 68(6), 19 (2017)

10. Szulkin, A., Weth, T.: The method of Nehari manifold. In: Handbook of Nonconvex Analysis and Applications, pp. 597-632. Int. Press, Somerville (2010)

Publisher's Note Springer Nature remains neutral with regard to jurisdictional claims in published maps and institutional affiliations. 